\title{
Design Tools for Small Implantable antennas
}

\author{
Ismael Vico Trivino ${ }^{1}$, Zvonimir Sipus ${ }^{2}$, Marko Bosilievac ${ }^{2}$, Anja K. Skrivervik ${ }^{1}$ \\ ${ }^{1}$ Microwave and Antenna Group, Ecole Polytechnique Fédérale de Lausanne, Switzerland \\ anja.skrivervik@epfl.ch \\ ${ }^{2}$ Faculty of Electrical Engineering and Computing, University of Zagreb, Croatia \\ marko.bosiljevac@fer.hr, zvonimir.sipus@fer.hr
}

\begin{abstract}
Implantable applications have been increasing in number in an exponential manner since the turn of the millennium. This is largely due to the increasing availability of ultra low power consumption electronics, enabling the emergence of healthcare services using implantable or swallowable sensors. Other fields of applications exist, e.g. in the domains of military, security or sports. A large number of antennas for these implantable or swallowable capsules have been designed, and can be found in the literature. They are however always presented for a specific capsule and application, and are thus very difficult to compare in terms of antenna characteristics.
\end{abstract}

In this paper, we show results obtained for simple canonical implantable radiation sources, obtained using a specifically developed numerical tool presented earlier. These canonical results will allow us to obtain some simple but useful figures of merit that enable an easy assessment of the quality of the radiation characteristic of a specific antenna design in a specific capsule

Index Terms-implantable antennas, limitations.

\section{INTRODUCTION}

Healthcare system are finding more and more applications for implantable or swallowable capsules, enabling the sensing of specific disease indicators (e.g. temperature or glucose levels or the monitoring of specific environments like the stomach or the colon). These capsules have all different shapes and dimensions, and all require a telemetry link in order to send the acquired data out of the host body to a base station. As far as capacity, frequency of communication and range are concerned, the requirements on these telemetry links are application dependent. However, they have in common the need for an extremely high efficiency in the use of the available power, as they are either powered remotely or make use of batteries where the time of life should be as long as possible [1]. Voltage scaling and dynamic power management have enabled to lower the power consumption of the electronics used in such devices; it should however be noted that the largest source of losses in such links is due to the dissipation undergone by the radiated electromagnetic fields in the lossy biological tissues hosting the capsules, and it can be shown that for medical implants , more than $95 \%$ percent of the power radiated by the antenna to communicate data outside the body is absorbed by the host body. Some of this absorption is unavoidable, as it is linked to the propagating wave traversing the lossy host medium. Another part, the losses due to the near field coupling to the biological medium is more difficult to assess, and to compare for different antennas.

In this contribution, we will show how to differentiate between unavoidable propagation losses inherent to the electromagnetic signal having to leave the lossy host body, and avoidable losses due to near field coupling between the electromagnetic fields in the vicinity of the implanted antenna and the lossy medium hosting it. To this aim, we will consider elementary EM sources placed in a spherical body phantom which can be multilayered in order to account for different tissues. This allows us to use a numerical tool based on a development in spherical waves which has been presented earlier $[2,3]$ and has the advantage of being very fast numerically. The conclusions drawn on these canonical cases will then be applied to analyze the performances of real implanted antennas, in which case commercial simulation codes will be used.

\section{RESULTS FOR CANONICAL SOURCES}

The spherical phantom (Fig. 1) and the numerical tool based on spherical wave expansion used for the analysis of the losses in canonical cases were described in [2-4].

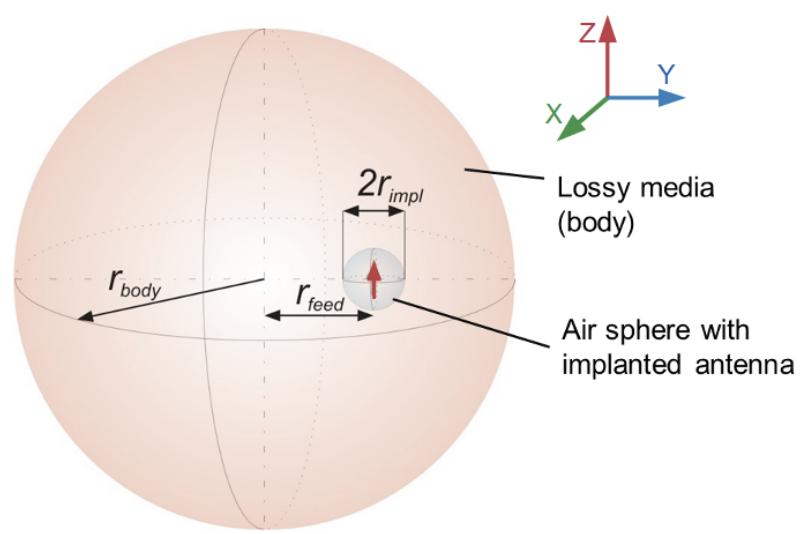

Fig. 1. Spherical single or multi layered phantom modelling parts of a human body. The source is an elementary electric or magnetic dipole (in red), and is surrounded by a lossless sphere [2].

Three interesting results obtained through this canonical model are recorded below [3]. The phantom used consists of a $18 \mathrm{~cm}$ diameter sphere, made of a homogeneous lossy material characterized by $\varepsilon_{r, b o d y}=43.5-\mathrm{j} 34.75$, which 
corresponds to the IEEE head model at $403 \mathrm{MHz}$ which is a standard frequency for medical telemetry.

Fig. 2 shows the total radiated power outside the phantom for three different elementary sources as a function of the position of the source inside the phantom. The orientation of the source is parallel to the surface of the phantom when it reaches the interface with free space. The radius of the air bubble enclosing the source is $0.1 \mathrm{~cm}$.

Fig. 3 shows the total power radiated outside the phantom for an electric source parallel to the phantom/free space interface, for different diameter of the air bubble enclosing the source, as a function of the source position in the phantom.

Fig.4 shows the total radiated power in the phantom reaching out of a sphere of radius $\mathrm{R}$ for an electric and $\mathrm{a}$ magnetic source placed at the centre of the phantom in an air bubble of radius $0.1 \mathrm{~cm}$.

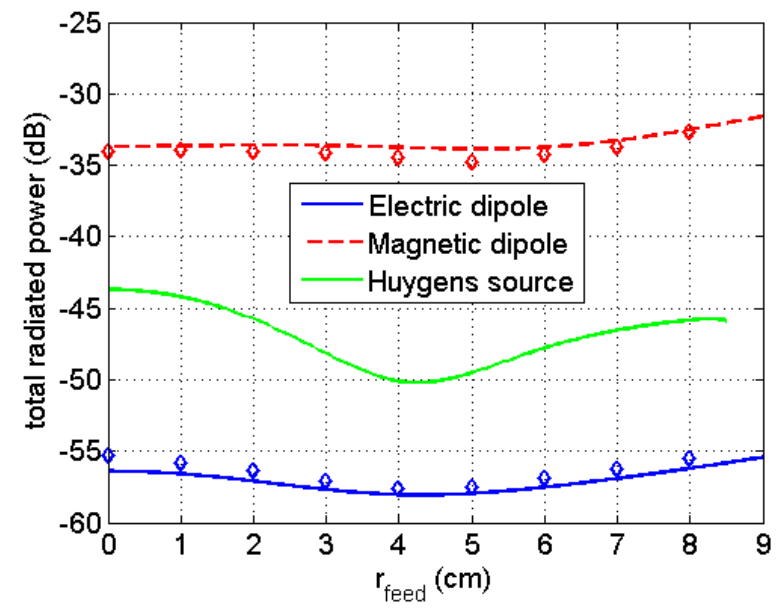

Fig. 2. Total radiated power as a function of source position in the phantom for three elementary sources. Solid and dashed lines - spherical mode approach; Diamonds - CST Microwave Studio.[3]

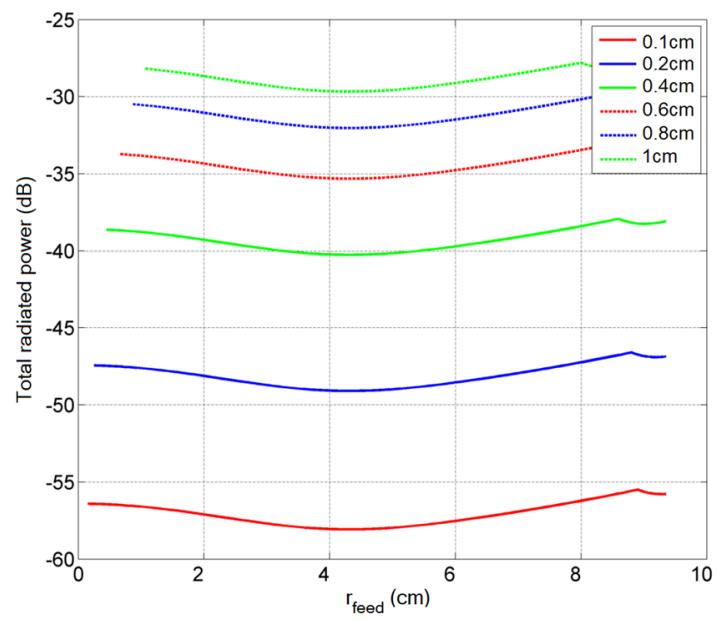

Fig. 3. Total radiated power reaching the free space for a parallel electric source as a function of source position and for different radii of teh lossless sphere around the source, at $403.5 \mathrm{MHz}$. [3]

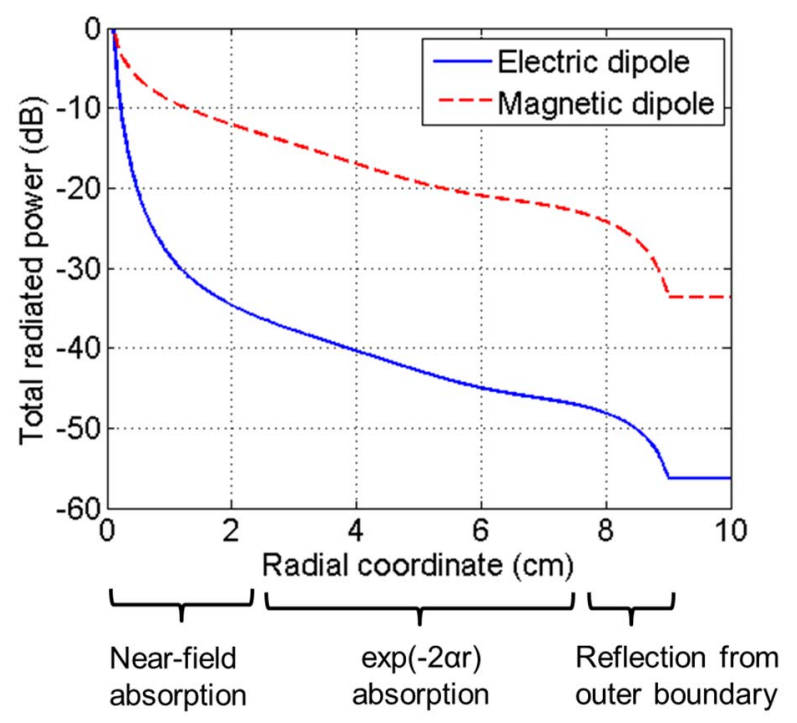

Fig. 4. Total radiated power in the phantom reaching out of a sphere of radius $\mathrm{R}$ (radial coordinate) for an electric and a magnetic source placed at the centre of the phantom at $403 \mathrm{MHz}$. [3]

Several interesting conclusions can be drawn from these results: First of all, we see that the amount of power reaching out of the lossy phantom does not depend primarily on the position of the source inside the phantom, but much more on the diameter of the lossless bubble enclosing the source and the nature of the source. The latter point is intuitively readily understood, as the phantom has dielectric and not magnetic losses, and near field coupling to the lossy tissue will be much more acute in the case of the case of an electric source, than for a magnetic source. Fig. 4 shows also clearly that the near field absorption is different depending on the type of source, but that the loss for the "propagating" region is similar for electric and magnetic sources.

\section{RESULTS FOR REAL ANTENNAS}

The conclusion we can draw from section II is clear: two elements have an impact on the radiation performance of an implanted antenna: the nature of the antenna (should be more magnetic than electric) and how the antenna designer managed to confine the near field area of the antenna in the lossless capsule containing the entire sensor. Thus, in order to compare two antenna designs for similar applications, the level of achievement of these two criteria should be compared. This can be done in several ways, one being the analysis of the wave impedance of the wave generated by the antenna inside the host body.

As an example, let us consider the case of the antenna of a real implanted capsule [5] compared to very small real but ideally matched dipole and loop antennas, as depicted in Figs. 5- 8. It has to be noted that the capsule was implanted in vivo, and showed excellent radiation performances as the reading range was larger than $14 \mathrm{~m}$ [5]. All antennas are placed in a phantom having a diameter of $9 \mathrm{~cm}$, and a homogeneous dielectric permittivity corresponds to the IEEE head model at $403 \mathrm{MHz}$ and $2.45 \mathrm{GHz}$. Indeed, the real 
capsule is dual frequency, the lower band being used for the data transmission and the ISM band for the wake up signal.

The total length of the dipole depicted in Fig. 5 is $0.44 \mathrm{~cm}$, which corresponds to $\lambda / 183$ at $403 \mathrm{MHz}$. The dipole is ideally matched using a lumped inductance in order to compensate for the capacitance. The diameter of the loop (see Fig. 6) is $0.4 \mathrm{~cm}$, and the antenna is ideally matched using a lumped capacitor. The dipole and the loop are placed in air bubbles having a diameter of $1 \mathrm{~cm}$ and $0.6 \mathrm{~cm}$ respectively. The PIFA used in the real existing and implanted capsule [5] is depicted in Fig. 8, along with the dimension of the implanted capsule.

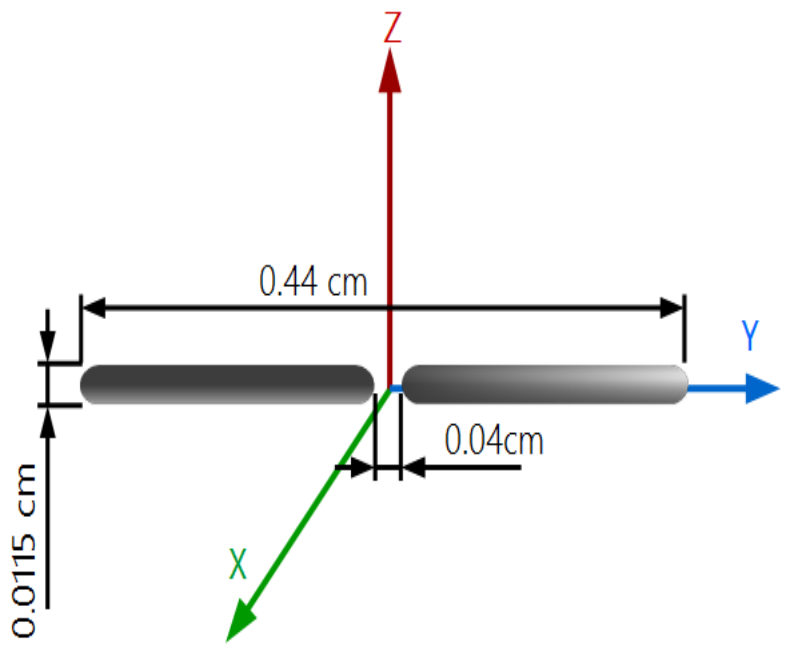

Fig. 5. Dimensions of the small dipole antenna. At the freqeuncy of 403 $\mathrm{MHz}$, its total length is $\lambda / 183$.

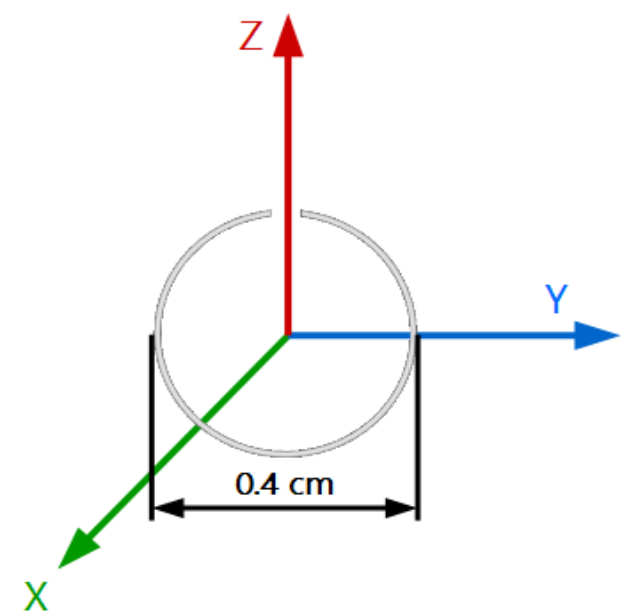

Fig. 6. Dimensions of the small loop antenna.

Fig. 7 shows the dimensions of the phantom and the position of the antennas in them. Please note that in this case the diameter of the phantom was shrunk to $9 \mathrm{~cm}$ for computer time reasons.
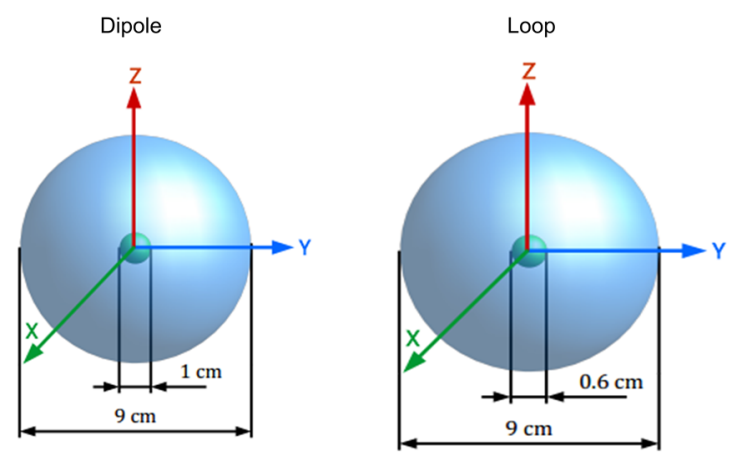

Fig. 7. Phantoms containg the real but small and ideally matched dipole and loop

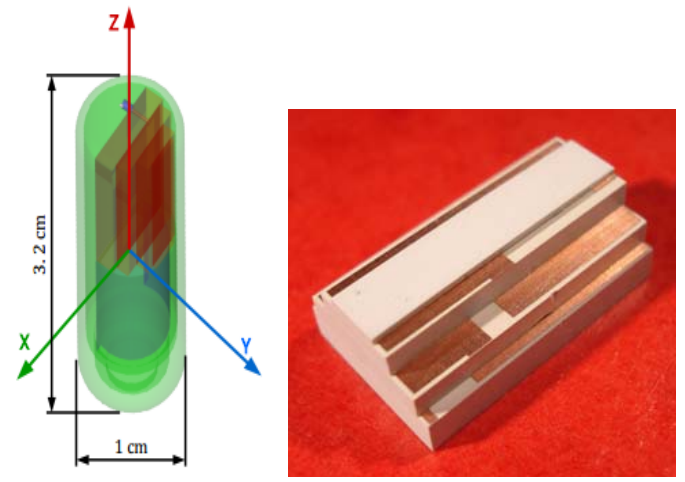

Fig. 8. Dimensions of implantable capsule and figure of the PIFA antenna used in this capsule.

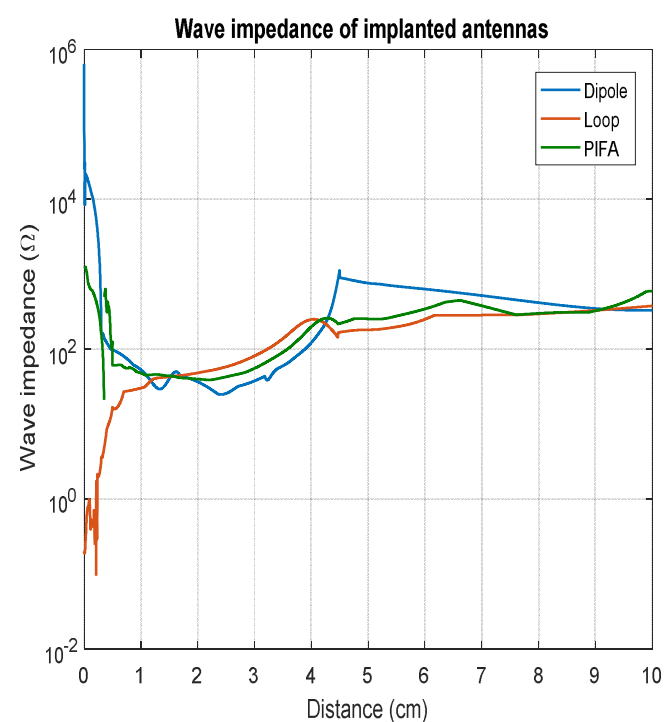

Fig. 9. Wave impedance for three different antennas at $403 \mathrm{MHz}$

Fig. 9 and 10 show the wave impendence as a function of the distance to the source due to these three antennas along the $\mathrm{x}$ axis for $403 \mathrm{MHz}$ and $2.45 \mathrm{GHz}$, respectively. The impedance is computed numerically by taking the transverse component of the $\mathrm{E}$ field divided by the transverse component of the $\mathrm{H}$ field. 


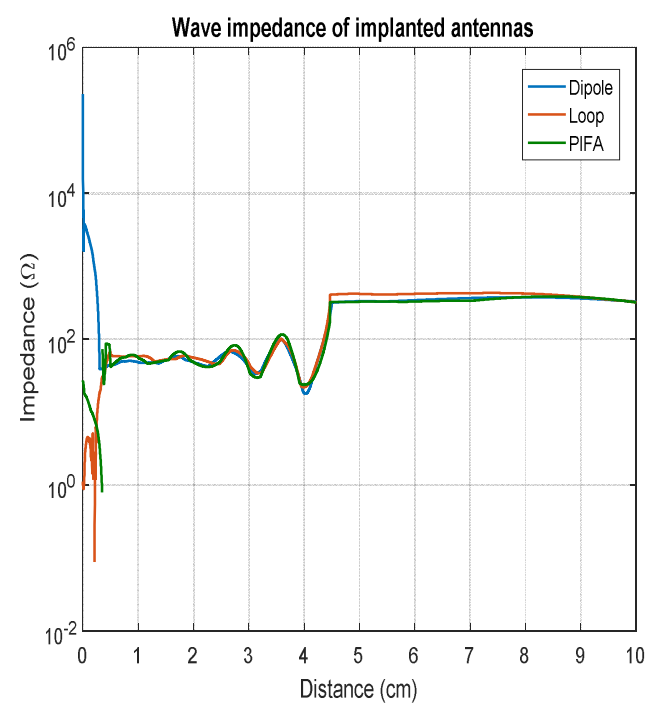

Fig. 10. Wave impedance for three different antennas at $2.45 \mathrm{GHz}$

For the lower frequency of $403 \mathrm{MHz}$, we see clearly that close to the sources, the wave due to the dipole is mostly electric, the wave due to the loop is mostly magnetic, while the wave due to the PIFA is more electric than magnetic, but still much less than for the dipole.

We see also that after a distance of $10 \mathrm{~mm}$, the wave impedance for the three antennas is more or less the same, which means that we have left the near field region and have reached the propagating region. The wave impedance then changes slowly as the wave reaches the phantom-air interface. This is due to the reflection at this interface.

For the higher frequency of $2.45 \mathrm{GHz}$, the dipole is again clearly electric close to the source and the loop magnetic, while the PIFA shows this time a more magnetic type of behavior. This stems from the fact that the arm of the PIFA forms a spiral (see Fig. 8), and that the inductance of this spiral becomes more important when the frequency increases. We see also that the near field region is in this case much smaller, and the propagating region is reached after about $6 \mathrm{~mm}$. The reflection due to the interface is also clearly observed.

Looking at Figs 9 and 10 helps to understand why the implant with the PIFA antenna worked well when it was implanted: As it is a PIFA, one would expect that its near field would be mainly of the electric type. This is not the case, due to the spiral of the arm of the PIFA, which compensates for the capacitances of the arm above ground. Moreover, as can be seen in [5], the ground is offset with respect to the arm of the PIFA, which again lowers the capacity of the latter. Finally, we can also note that most of the near field region is compressed in the lossless region of the capsule, showing that this antenna is close to optimum.

\section{CONCLUSIONS}

In order to assess the overall efficiency of an implantable or swallowable antenna, the nature of the antenna and the possibility to confine the antenna near field in a lossless region will be key parameters. A way to assess the quality of the radiation characteristics of a real antenna is to analyze its wave impedance numerically inside the lossy medium, as was shown on the practical case of an antenna for a real implantable capsule.

\section{REFERENCES}

[1] P.S. Hall, Y. Hao, Y.I. Nechayev, A Alomainy, CC Constantinou, C Parini et al. "Antennas and propagation for on-body communication systems", Antennas and Propagation Magazine, IEEE 49 (3), 2007, pp. 41-58.

[2] M. Boslijevac, Z. Sipus, A.K. Skrivervik, "Propagation in Finite Lossy Media: an Application to WBAN", IEEE Antennas and Wireless Propagation Letters, Year: 2015, Volume: 14, PP1546 1549.

[3] M. Bosiljevac, Benjamin Fuchs, A.K. Skrivervik and Z. Sipus, "Study of wearable WBAN antenna properties based on spherical body model", EuCAP 2016, Davos, April 10-15, pp 1-3.

[4] Anja K. Skrivervik, Marko Bosiljevac, and Zvonimir Sipus, "Propagation Considerations for Implantable Antennas", EuCAP 2017, Paris, March 19-24, pp 1573-1577.

[5] F. Merli, L. Bolomey, F. Gorostidi, B. Fuchs, J. F. Zürcher, Y. Barrandon, E. Meurville, J. R. Mosig, and A. K. Skrivervik, "Example of data telemetry for biomedical applications: an in vivo experiment," IEEE Antennas and Wireless Propagatoin Letters, vol. 11, num. part II, p. 1650-1654, 2012. 